\title{
Responsibility, culpability, and parental views on genomic testing for seriously ill children
}

\author{
Janet Malek, PhD (i) ${ }^{1}$, Stacey Pereira, $\mathrm{PhD}{ }^{1}{ }^{1}$, Jill O. Robinson, $\mathrm{MA}^{1}$, Amanda M. Gutierrez, BA ${ }^{1}$, \\ Melody J. Slashinski, MPH, PhD², D. Williams Parsons, MD, PhD ${ }^{3,4}$, Sharon E. Plon, MD, PhD ${ }^{3,4}$ and \\ Amy L. McGuire, JD, PhD ${ }^{1}$
}

Purpose: We describe parental perceptions of and experiences with genomic sequencing (GS) in the care of seriously ill children. Understanding parents' perspectives is vital for clinicians caring for children, given the uptake of genomic technologies into clinical practice.

Methods: Longitudinal, semistructured interviews were conducted with parents of pediatric cancer patients who underwent exome sequencing (ES) as a part of the BASIC3 study. Interviews were conducted at baseline, one to eight months after results disclosure, and approximately one year after disclosure. Using thematic qualitative analysis, parent interviews were coded with both inductive and deductive approaches.

Results: Before receiving genomic information, parents indicated that they saw ES as something responsible parents would agree to if their child had cancer. Some parents talked about the possibility of sequencing affecting feelings of culpability for their child's cancer, worrying that they passed on a cancer-causing gene or made parenting decisions that caused the disease. However, after receiving their child's ES results many reported feeling relieved of guilt and worry, and felt they had fulfilled parental duties by agreeing to ES for their child.

Conclusion: These results reveal a layer of meaning that parents associate with GS that may inform clinicians' approach to care.

Genetics in Medicine (2019) 21:2791-2797; https://doi.org/10.1038/s41436019-0570-6

Keywords: guilt; blame; responsibility; genomic sequencing; parent perspectives

\section{INTRODUCTION}

Genome-scale sequencing (GS) is increasingly being integrated into pediatric clinical care. ${ }^{1,2}$ Both clinicians and parents hope that this information will be used to improve diagnosis and/or create safer, more effective treatment plans for children with serious medical conditions. Beyond these shared clinical aims, GS has the potential to generate information that may raise special considerations for parents facing decisions about the use of this technology. It is therefore vital for clinicians caring for seriously ill children to have a thorough, nuanced understanding of the meaning that GS results may have for parents. Our team has previously reported on the clinical, psychological, and pragmatic utility that parents associate with GS. ${ }^{3}$ Here we explore how a parent's role as a child's progenitor and caretaker may shape her views and experiences.

There is limited empirical work describing parental views on GS that directly addresses this question. One study found that parents of children with rare genetic disorders described a duty to do everything possible to understand their child's condition, including GS. ${ }^{4}$ Similarly, in other research, parents of children with undiagnosed disorders felt an obligation to try GS to obtain a diagnosis ${ }^{5}$ and gather information about any other genetic risks. ${ }^{6}$ These results suggest that parents feel a duty to use GS as part of a general parental obligation to explore all possible ways to help their child.

Professional caregivers, ${ }^{7}$ parents of children with autism, ${ }^{8}$ and parents of children with epilepsy ${ }^{9}$ have speculated that genomic screening results could bring about feelings of parental guilt and blame as well as the possibility of relief from guilt. Other studies have confirmed that in some cases parents do respond to a child's genetic diagnosis with feelings of guilt or attributions of blame. ${ }^{10-12}$ On the other hand, parents may have concerns that other parenting decisions they made led to their child's illness ${ }^{13}$ and believe that a genetic diagnosis could relieve these feelings of culpability. ${ }^{14}$ In two studies with parents of children with disabilities, participants reported feeling absolved of guilt and blame upon ${ }^{1}$ Center for Medical Ethics and Health Policy, Baylor College of Medicine, Houston, TX, USA; ${ }^{2}$ School of Public Health \& Health Sciences, University of Massachusetts, Amherst,
MA, USA; ${ }^{3}$ Department of Pediatrics, Baylor College of Medicine, Houston, TX, USA; ${ }^{4}$ Texas Children's Cancer Center, Texas Children's Hospital, Houston, TX, USA. Correspondence: Janet Malek (janet.malek@bcm.edu) 
learning that there was a genomic explanation for their child's condition. $^{15,16}$

In previous literature, these possible responses are typically described as one of many risks or potential benefits to be disclosed during an informed consent process. This paper uses the results from interviews with parents of pediatric cancer patients before and after receiving germline and tumor exome sequencing (ES) results to develop a more holistic understanding of how parenthood shapes perceptions about GS information for parents of children with cancer. We then draw connections between our results and those from other studies to defend a broader thesis about how parents may think about genomic testing in the context of clinical care more generally. An in-depth understanding of how one's role as a parent affects perceptions of or experience with GS is vital for clinicians who are increasingly incorporating this technology into routine practice, informing best practices for offering GS and returning its results to parents of seriously ill children.

\section{MATERIALS AND METHODS}

The Baylor Advancing Sequencing in Childhood Cancer Care (BASIC3) study evaluated the integration of germline and tumor (if a sample was available) ES results into the clinical care of pediatric patients with newly diagnosed solid tumors. The BASIC3 study enrolled 287 patients at Texas Children's Cancer Center (TCCC) in Houston, Texas between August 2012 and June 2016. Participation in the study included germline ES for all patients and also tumor sequencing when possible ( $80 \%$ of patients enrolled). Informed consent was obtained from all subjects. The BASIC3 study consenting, sequencing results, and disclosure processes have been reported elsewhere. ${ }^{17,18}$ The study was approved by the Institutional Review Board (IRB) of Baylor College of Medicine as the IRB of record for Texas Children's Hospital (TCH).

Each patient's primary oncologist and a study genetic counselor disclosed the germline and, if applicable, tumor ES results to the family. The categories of germline results reported included diagnostic findings and variants of uncertain significance (VUS) related to the child's phenotype (cancer or other diseases), medically actionable secondary findings, a small number of pharmacogenomic findings, and carrier status for recessive disorders (if the parent opted to receive their child's carrier information). Results pertaining to adult-onset conditions that were unrelated to the patient's current medical status (other than those deemed medically actionable) were not specifically analyzed or disclosed as a part of this study. Families were informed that it was unlikely that the patient's ES results would change the patient's current treatment.

Parents enrolled in the BASIC3 study participated in semistructured interviews in their language of preference (English or Spanish) at three time points: baseline ("predisclosure"), one to eight months after disclosure ("postdisclosure"), and approximately one year after disclosure ("follow-up"). Interview questions were open-ended, inviting participants to reflect on their experience of having a child with cancer, decision-making about treatment, expectations of GS, feelings about the results, and responses to the information. Most interviews involved only one parent, typically the patient's mother. Interviews were conducted at a convenient time and place for the family, often in the clinic at the time of clinically indicated visits, and lasted between 15 and 90 minutes. The family was compensated \$25 after completing each interview. To meet the longitudinal goal of 25 follow-up interviews, we oversampled for predisclosure $(n=64)$ and postdisclosure interviews $(n=33)$. Figure 1 shows reasons for attrition throughout the interview portion of the study. Interviews included in this analysis were conducted between August 2012 and February 2017.

Parent interviews were audio-recorded and transcribed verbatim. Interview transcripts were reviewed for accuracy and uploaded to ATLAS.ti (v 7.5.11, Scientific Software Development, GmbH, Berlin, Germany), a qualitative data management software program. All potentially identifying information was removed. Parents are only referred to by their study identification number, noted in parentheses after each quotation. Using thematic qualitative analysis, we first used a deductive coding approach to create initial codes based on main research questions. ${ }^{19}$ An inductive approach was then utilized to create additional codes based on themes identified in the interviews. Parental perceptions of sequencing utility and the expectations of families and study oncologists of the impact of receiving patients' ES results have been described elsewhere. ${ }^{3,20}$

\section{RESULTS}

Parents of children with cancer were interviewed before and after their child's germline and tumor ES results were returned to them with the aim of understanding their experience with this increasingly used technology.

\section{Description of participants}

Table 1 shows the demographics of parents $(n=64)$ who completed at least one interview with a total of 122 interviews analyzed. Most parents $(87.5 \%)$ were female and mothers of the patient $(82.8 \%)$ and many self-identified as Hispanic or Latino (42.2\%), consistent with our clinic population. About half of the participants were not college graduates (52.0\%) and a similar proportion (52.4\%) have an annual household income under $\$ 50,000$.

\section{Results overview}

Throughout our interviews, we found that the participants' role as a parent shaped their expectations about and experiences with ES. In particular, themes emerged related to their duty to make decisions that will be good for their child and contributions to their child's genetic makeup. Although interviewers did not ask questions specifically designed to explore this aspect of the study, 39/64 (61\%) parents spontaneously raised issues related to at least one of these two themes: responsibility $(12 / 64 ; 19 \%)$ and culpability 


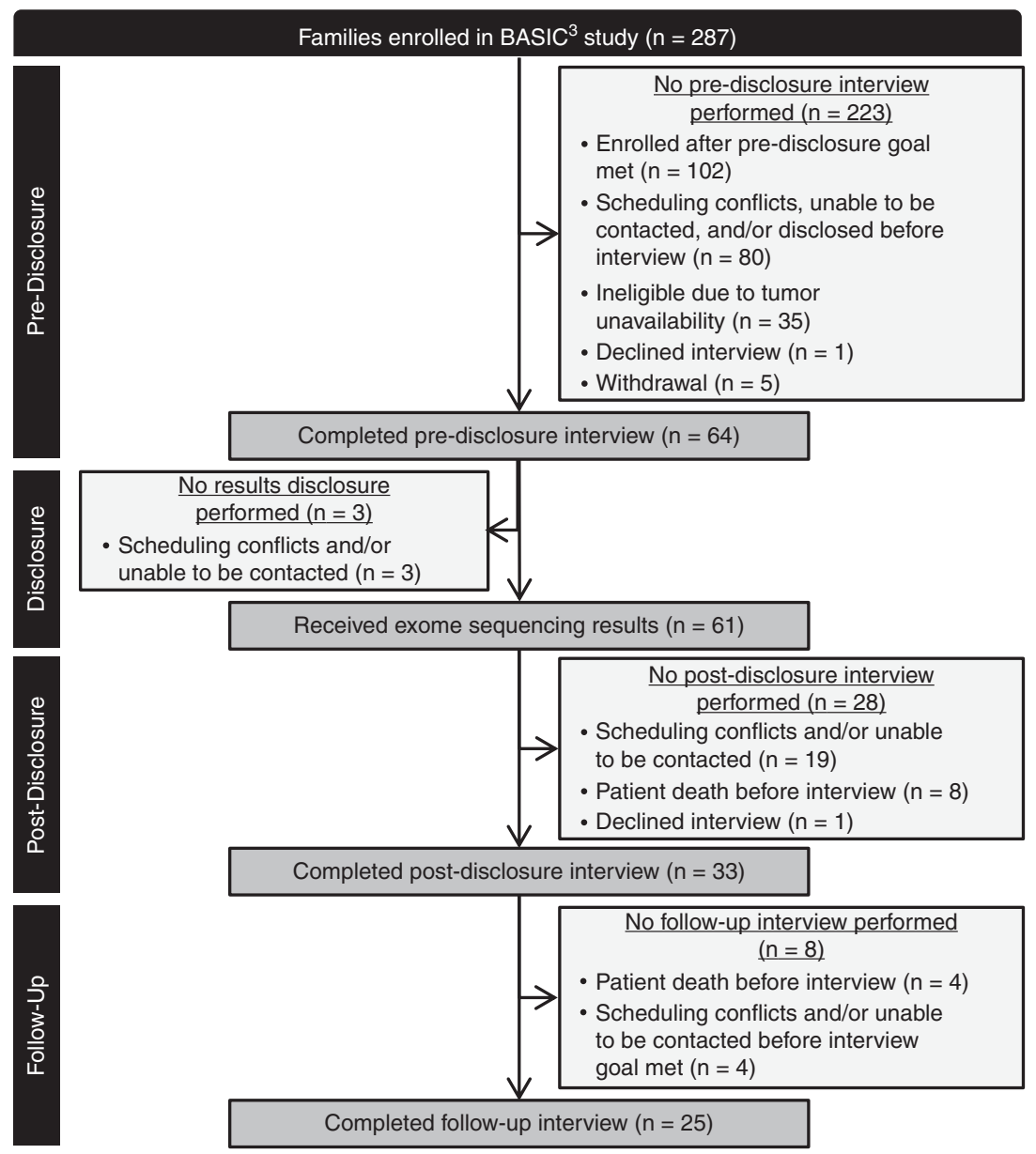

Fig. 1 BASIC3 study consort diagram.

Table 1 Parent demographics ${ }^{\mathrm{a}}$

$$
N=64^{\text {b }}
$$

\begin{tabular}{lc}
\hline $\begin{array}{l}\text { Gender } \\
\text { Female }\end{array}$ & $56(87.5)$ \\
\hline Male & $8(12.5)$ \\
\hline Relation to patient & $53(82.8)$ \\
\hline Mother & $9(14.1)$ \\
\hline Father & $2(3.1)$ \\
\hline Legal guardian & \\
\hline Race/ethnicity & $27(42.2)$ \\
\hline Hispanic or Latino & $25(39)$ \\
\hline Non-Hispanic or Latino, White & $12(18.8)$ \\
\hline Non-Hispanic or Latino, Other Race
\end{tabular}

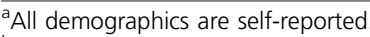

bunless otherwise noted.

'Non-Hispanic or Latino, Other Race includes Asian (4), Black or African American (8), two or more races (2), declined, or NA (9).

$(31 / 64 ; 48 \%)$. The prevalence of these responses suggests that these considerations were salient issues for many participants.

Parents indicated that they saw ES as something a responsible parent of a child with cancer would do if offered. "Responsibility" in this sense refers to the participants' intentions to meet the set of expectations they associate with parenthood. It is worth noting that this concept is slightly different from the genetic responsibility described by others, ${ }^{21}$ referring to the obligation a person may have to understand and manage genetic risk information.

Parents also described considerations about culpability associated with genomic information. They talked about "guilt," describing their own feelings resulting from the concern that they themselves had done something wrong. Several parents also made observations about "blame," referring to the possibility that others would attribute fault to them for their child's cancer. They worried about receiving sequencing results that would generate guilt and blame for passing on a cancer susceptibility gene or for having made poor parenting decisions. After receiving their child's results, many parents reported that they were relieved of this guilt and worry.

\section{Responsible parenthood}

Many parents saw choosing ES to be a way of fulfilling parental obligations. Parents felt that a requirement of being a 
responsible parent was doing everything possible to help their child. Some saw ES as an opportunity to gain additional information to help them understand and better prepare for their child's illness, while others simply felt that in choosing ES, regardless of the results, they were doing everything possible to fulfill their parental obligations.

"As I said, we wanted to make sure that as a responsible parent, we are doing everything in our knowledge and understanding, to make sure that we know about everything that our child [has] gone through and is going to go through, so that we are prepared for everything that's out there." (Post 350, no medically actionable, cancer-related, or tumor variants)

"Hopefully they'll be able to find something, I guess. I just want to make sure we did everything. If it does, it does. I just wanted to make sure we did everything." (Pre 265)

Others interpreted their child's illness as their own failure to fulfill their parental obligations. They described preventing or discovering the cancer early to be part of their responsibility as a good parent and saw ES as a way of potentially mitigating and avoiding this failure in the future.

"It's also important because maybe like I said, I can head something off before it's started. I feel very guilty that he got this because I feel like it's my job. I should have caught it. And even though there was nothing I could have done about it, it's still-you know, you're his mother and you should know these things." (Pre 268)

\section{Concern about culpability for cancer: inheritance}

In predisclosure interviews, parents identified culpability for possibly passing on genetic variants that caused their child's cancer as a consideration when deciding to do ES. Some simply questioned how they would react to this type of information, wondering whether they would feel guilty and whether others would blame them if the results showed that they had passed on a cancer-causing gene.

"I mean there's part of you that-you know if you thinkhopefully there won't be a guilt associated with that. Would it have come from me? Would it have come from my side? You know. But at the same time I think-I don't think we can think like that. If that's not information we knew ahead of time. You know?" (Pre 226)

PARENT 1: “(laughs) Oh he wants to-no, he's the type of person-'Well, it's your fault.' That's why he wants it, so he can lay it on. 'Well, you know, because this, this, and this, you made it because she has this now.' That's what he wants. He wants to know so he can blame it on whoever. (laughs)

PARENT 2: No, I ain't going to be playing the blame game.

PARENT 1: Yes he is. Yeah, he is.” (Pre 256)

A few parents worried about how they would manage the guilt associated with the potential of finding that they had passed on a cancer-causing gene to their child. In particular, one parent expressed how "intellectually" she wanted the information but was concerned about the emotional impact of receiving such a result, parsing the meaning of the ES information for her and her relationship with her child into two distinct layers.

“As much as I want the knowledge-let's say I do find out I'm a carrier, yeah, I'm not going to have more kids, how do I deal emotionally with that? Then that starts-the whole emotional processing of it. Is like, the guilt, now it's my fault, you know? How do you deal with that? You know?...it's scary on an emotional level how do you deal with it." (Pre 232)

Other parents focused on the possibility of learning that the cancer was not caused by an inherited gene and were optimistic that this would exculpate them of responsibility for their child's cancer.

"But I see it in this way that-you know-there might be a hope. There might be a good answer for us that you know, it is not us. It's just a bad luck." (Pre 235)

"I'm really just hoping-you know-he didn't get it-you know-anywhere from me." (Pre 345)

\section{Concern about culpability for cancer: parenting decisions}

Passing on an inherited gene variant was not the only source of culpability that parents felt for their child's cancer. Many parents expressed concern about whether they had done something, or not done something, while raising their child that had caused the cancer. Finding a genetic cause for the cancer was perceived by some as a way to relieve this parental guilt by providing assurance that there was nothing they could have done differently to prevent their child's cancer, and thus, they had not failed in their roles as responsible parents.

"Like it would be like, oh it's a genetic factor, there's nothing you can do to prevent it. It would make me feel a whole lot-in a way I hate to say it-but a whole lot better. And to know that I caused it all of the fact that it's 
something I did, that I gave her to eat or something that she ate or something that-maybe because I bought her a cell phone too early in her life...it would help me in my way knowing that I didn't fail in that way, you know." (Pre 292)

"There's one instance when she was five when she fell, and she hit her head...I didn't think of having it checked, but this scenario plays in my head all the time. I'm like man, what if that caused it, and I should have taken her to the doctor? Even if I had taken her anyways maybe they would have seen that, and it could have prevented all of this. I think if I felt that it was something that was like out of my control [like a genetic cause] then maybe it wouldn't be like a guilt factor there." (Pre 270)

\section{Relief from guilt and worry about culpability after ES disclosure}

Despite concerns that were raised predisclosure, in postdisclosure interviews most parents reported feeling relieved from worries about culpability after receiving their child's ES results. None of the four parents interviewed at baseline who were found to have passed a cancer susceptibility gene on to their child participated in postdisclosure interviews. One family was not interviewed because the patient died, two families were lost to follow-up, and one was not contacted because our Spanish-speaking interviewer was not available. As a result, our data do not include parental reactions to positive germline testing.

For the parents interviewed, the results allayed concerns that they did or failed to do something that caused their child's cancer. Interestingly, parents felt this relief regardless of whether the ES results were negative, positive for tumor pathogenic variants, or positive for secondary, medically actionable findings.

Many parents with negative results described the relief, and even joy, they felt upon finding out that they had not passed on a genetic variant that caused their child's cancer. To them, this meant that they were not to blame, even though they had been informed that ES does not detect all types of cancer susceptibility variants.

"It was a relief. I mean, they had already told us that they were more than like-more than positive that it wasn't a genetic type of tumor. So that was good. But then just to hear them actually say it from all the tests was good...so that made me feel-feel really good, you know, not to be blamed." (Post 346, positive tumor pathogenic variant)

"There was a lot of information. But I just-I think my mind just stuck on that and it was just sticking right there, that it wasn't us...It wasn't us. (laughing) I was just-I was very joyful." (Post 281, no medically actionable, cancerrelated, or tumor variants)

\begin{abstract}
"Makes it easier because it sort of helps me with 'I should have known something was wrong a long time ago' kind of mentality that I think parents go through. But it also makes me realize that there's absolutely nothing I could have done about it and the genetic people don't even know why he got it. So, it's not like it was something I missed. No one would have known that he was going to come down with this based on genetic testing. So, that helps, sort of, I think." (Post 254, no medically actionable, cancer-related, or tumor variants)
\end{abstract}

Parents who received results that showed tumor-specific or somatic variants without germline variants also felt relief. They interpreted these findings to mean that they had done nothing wrong as parents. The tumor pathogenic variant meant to them that they had not failed in their responsibilities as good parents, and as such, it actually provided reassurance to them.

"Knowing that there was nothing that we did wrong. It was not us. We weren't really parents that did anything wrong or whatever. It was just very environmental and something in his body that didn't... Cell that didn't work correctly. It decided to become... What, as they told the kids, a bad cell...Yeah, so. It was nice. Just a big relief." (Follow-Up 346, positive tumor pathogenic variant)

\section{DISCUSSION}

Our findings align with reports about the experience of parents of children with a variety of other serious illnesses, suggesting that they are not unique to the context of childhood cancer. ${ }^{4,10-12,15,16}$ Furthermore, they offer confirmatory evidence for data generated using hypothetical scenarios in other studies. ${ }^{7-9}$ The fact that these considerations were raised spontaneously and consistently by the majority of parents $(61 \%)$ we interviewed indicates that they are of substantial and common concern. From our results and those of others emerges a picture of the impact parenthood can have on experiences with genomic information. They demonstrate that GS has an additional layer of meaning beyond clinical utility for parents of seriously ill children that might not be immediately apparent to clinicians. That is, rather than describing concerns about culpability, possible relief from guilt, and fulfillment of parental responsibilities as risks and benefits of the use of GS technology, they should be understood as considerations that shape the ways in which other risks and benefits of GS are evaluated by parents of seriously ill children. This layer of meaning may consciously or unconsciously affect parents' decisions about using GS, color their experience with the technology, and influence their interpretation of the results. 
Understanding the meaning that GS may have for parents enables clinicians to participate more effectively in shared decision-making about the use of this technology. Many parents we interviewed felt that they had an ethical obligation to consent for ES because that is what they believed a responsible parent would do. If offered, parents may feel morally compelled to accept ES. It may be appropriate for clinicians to be particularly careful to discuss the optional nature of the decision, particularly for new genomic technologies associated with research studies, when raising the possibility given this insight. Others anticipated that ES would find a cause for the cancer, absolving them of worries that they had done something to bring it about. This suggests that parents may choose to undergo GS at least in part to gather exculpatory evidence "proving" that they did not fail to fulfill their parental obligation to protect their child. An awareness of how these considerations might affect a parent's decision-making can help clinicians decide when to raise the possibility of GS, offer appropriate information about the technology, and set reasonable parental expectations for its results.

Of particular interest is the question of how clinicians should account for or accommodate parental expectations and reactions, some of which may not be supported by scientific realities, with respect to culpability and responsibility. For example, identification of a tumor pathogenic variant may not offer exculpatory information about either the genetic basis of the child's cancer although in some cases specific somatic variants make genetic susceptibility less likely. GS results do not offer insight into whether some action or inaction on the part of the parent caused the child's cancer; however, several parents believed this information would assuage concerns that it had. Parents also anticipated feeling guilty about transmitting a cancer-causing gene although they could not have prevented it. Finally, parents described relief from getting a negative inheritance report despite having been informed at multiple time points that ES does not identify all genetic causes of cancer. Similarly, for some parents, the lack of an explanation allowed them to attribute their child's cancer to plain bad luck. These reports raise questions about whether these perceptions could be managed by an enriched informed consent process.

Our findings can also inform the process of disclosing ES results to parents and patients. Clinicians attending to the full range of meaning that these results may have for parents can frame the disclosure in a way that minimizes parental concerns about culpability. They can better understand the questions parents may have and know to offer counseling and support resources when appropriate.

\section{Limitations}

As a qualitative study based on interviews with open-ended questions, these data do not offer information about the prevalence of parents' various perspectives. Second, as our study population consisted solely of parents of pediatric cancer patients, these findings may not be generalizable to parents of children in other populations. Third, our findings may reflect the fact that our participant pool was heavily weighted toward mothers (82\%). Further work would need to be done to evaluate whether our conclusions apply similarly to both mothers and fathers.

Only a minority of the parents interviewed at the postdisclosure time point had a child who had significant positive findings, including 11 tumor pathogenic variants, two secondary medically actionable findings, and one cancer susceptibility gene found in a child who had been adopted. Although 11 BASIC3 participants had a germline finding indicating increased cancer risk, ${ }^{18}$ only 4 of these parents participated in the predisclosure interviews and none responded to requests for postdisclosure interviews. Access to these parents' perspectives might have changed the tone of our results, revealing a more balanced picture of the pros and cons of GS. However, the absence of these data does not undermine the paper's thesis that these technologies have an additional layer of meaning associated with responsibility and culpability for many parents of seriously ill children. If anything, these considerations are likely to be more salient for parents who passed genetic susceptibility to cancer on to their children. The fact that these themes arose so consistently in interviews with parents who received largely negative results emphasizes their importance to parents' decision-making and experience.

\section{Conclusion}

To these parents of seriously ill children, particularly those who were newly diagnosed, ES and its results had significance that clinicians may not readily anticipate. This layer of meaning may affect both parents' decision-making about ES and their experience with receiving their child's results. Parents may view GS both as a means of fulfilling parental duties and gaining reassurance that they haven't failed to fulfill those duties in the past. These insights may help clinicians caring for all children incorporate GS more ethically and effectively into their practice.

\section{ACKNOWLEDGEMENTS}

We thank Robin Raesz-Martinez, study coordinator; Sarah Scollon and Katie Bergstrom, study genetic counselors; and Uma Ramamurthy and her team at the Baylor College of Medicine Institute for Clinical and Translational Research. We also appreciated Larry McCullough's foundational contributions to this project. Finally, we offer particular gratitude to the oncologists and parents who participated in this study. The BASIC3 study is a Clinical Sequencing Exploratory Research (CSER) program project supported by the National Human Genome Research Institute and the National Cancer Institute (1U01HG006485).

\section{DISCLOSURE}

Baylor College of Medicine and Miraca Holdings Inc. have formed a joint venture with shared ownership and governance of the Baylor Genetics Laboratory, which performs exome sequencing. S.E.P. serves on the Scientific Advisory board of Baylor Genetics Laboratory. The other authors declare no conflicts of interest. 
Publisher's note: Springer Nature remains neutral with regard to jurisdictional claims in published maps and institutional affiliations.

\section{REFERENCES}

1. Bowdin S, Gilbert A, Bedoukian E. Recommendations for the integration of genomics into clinical practice. Genet Med. 2016;18:1075.

2. Rahimzadeh V, Schickhardt C, Knoppers BM, et al. Key implications of data sharing in pediatric genomics. JAMA Pediatr. 2018;172:476-481.

3. Malek J, Slashinski MJ, Robinson JO, et al. Parental perspectives on whole-exome sequencing in pediatric cancer: a typology of perceived utility. JCO Precis Oncol. 2017;1:1-10.

4. Sapp JC, Dong D, Start C, et al. Parental attitudes, values, and beliefs toward the return of results from exome sequencing in children. Clin Genet. 2014;85:120-126.

5. Rosell AM, Pena LD, Schoch K, et al. Not the end of the odyssey: parental perceptions of whole exome sequencing (WES) in pediatric undiagnosed disorders. J Genet Couns. 2016;25:1019-1031.

6. Anderson JA, Meyn MS, Shuman C, et al. Parents perspectives on whole genome sequencing for their children: qualified enthusiasm? J Med Ethics. 2017:43:535-539.

7. Hens K, Peeters H, Dierickx K. Genetic testing and counseling in the case of an autism diagnosis: a caregivers perspective. Eur J Med Genet. 2016; 59:452-458.

8. Tabor HK, Brazg T, Crouch J, et al. Parent perspectives on pediatric genetic research and implications for genotype-driven research recruitment. J Empir Res Hum Res Ethics. 2011;6:41-52.

9. Shostak S, Zarhin D, Ottman R. What's at stake? Genetic information from the perspective of people with epilepsy and their family members. Soc Sci Med. 2011;73:645-654.

10. James CA, Hadley DW, Holtzman NA, Winkelstein JA. How does the mode of inheritance of a genetic condition influence families? A study of guilt, blame, stigma and understanding of inheritance and reproductive risks in families with X-linked and autosomal recessive diseases. Genet Med. 2006;8:234-242.

11. Reiff M, Giarelli E, Bernhardt BA, et al. Parents' perceptions of the usefulness of chromosomal microarray analysis for children with autism spectrum disorders. J Autism Dev Disord. 2015;45:3262-3275.

12. Clarke A. Anticipated stigma and blameless guilt: mothers' evaluation of life with the sex-linked disorder, hypohidrotic ectodermal dysplasia. Soc Sci Med. 2016:158:141-148.

13. Matteo $B$, Pierluigi $B$. Descriptive survey about causes of illness given by the parents of children with cancer. Eur J Oncol Nurs. 2008;12:134-141.

14. Krabbenborg L, LELM Vissers, Schieving J, et al. Understanding the psychosocial effects of WES test results on parents of children with rare diseases. J Genet Couns. 2016;25:1207-1214.

15. Stivers $T$, Timmermans $S$. The actionability of exome sequencing testing results. Sociol Health Illn. 2017;39:1542-1556.

16. McLaughlin J, Clavering EK. Questions of kinship and inheritance in pediatric genetics: substance and responsibility. New Genet Soc. 2011; 30:399-413.

17. Scollon $\mathrm{S}$, Bergstrom $\mathrm{K}$, Kerstein RA, et al. Obtaining informed consent for clinical tumor and germline exome sequencing of newly diagnosed childhood cancer patients. Genome Med. 2014;6:69. https://doi.org/ 10.1186/s13073-014-0069-3. Accessed 7 Jun 2019.

18. Parsons DW, Roy A, Yang $Y$, et al. Diagnostic yield of clinical tumor and germline whole-exome sequencing for children with solid tumors. JAMA Oncol. 2016;2:616.

19. Creswell JW. Qualitative inquiry and research design: choosing among five approaches. 2nd ed. Thousand Oaks, CA: Sage Publications; 2012.

20. McCullough LB, Slashinski MJ, McGuire AL, et al. Is whole-exome sequencing an ethically disruptive technology? Perspectives of pediatric oncologists and parents of pediatric patients with solid tumors. Pediatr Blood Cancer. 2016;63:511-515.

21. Hallowell N. Doing the right thing: genetic risk and responsibility. Sociol Health IIIn. 1999;21:597-621. 